\title{
An Extended Linkage Map for Watermelon Based on SRAP, AFLP, SSR, ISSR, and RAPD Markers
}

\author{
A. Levi ${ }^{1}$ and C.E. Thomas \\ USDA, ARS, U.S. Vegetable Laboratory, 2700 Savannah Highway, Charleston, SC 29414 \\ T. Trebitsh and A. Salman \\ Department of Life Sciences, Ben-Gurion University of the Negev, Beer-Sheva, 84105, Israel \\ J. King and J. Karalius \\ Seminis Vegetable Seeds, 37437 State Highway 16, Woodland, CA 95695
}

M. Newman

USDA, ARS, Plant Genetic Resources and Conservation Unit, 1109 Experiment Street, Griffin, GA 30223-1797

O.U.K. Reddy

Department of Biology, West Virginia State University, Institute, WV 25112-1000

Y. Xu

National Engineering Research Center for Vegetables, Beijing 100089, China

\author{
X. Zhang \\ Syngenta Seeds, 21435 Road 98, Woodland, CA 95695
}

AdDitional IndeX words. Citrullus lanatus, Citrullus colocynthis, genetic mapping, DNA markers, vegetable breeding

\begin{abstract}
Seventy-one amplified fragment length polymorphism (AFLP), 93 sequence related amplified polymorphism (SRAP), and 14 simple sequence repeat (SSR) markers were used to extend an initial genetic linkage map for watermelon [Citrullus lanatus (Thunb.) Matsum. \& Nakai]. The initial map was based on 151 randomly amplified polymorphic DNA (RAPD) and 30 and inter-simple sequence repeat (ISSR) markers. A testcross population previously used for mapping of RAPD and ISSR markers was used in this study: \{plant accession Griffin 14113 [C. lanatus var. citroide (L.H. Bailey) Mansf.] $x$ the watermelon cultivar New Hampshire Midget (C. lanatus var. lanatus) $\}$ x PI 386015 [C. colocynthis (L.) Schrad.]. The linkage map contains 360 DNA markers distributed on 19 linkage groups, and covers a genetic distance of $1976 \mathrm{cM}$ with an average distance of $5.8 \mathrm{cM}$ between two markers. A genomic DNA clone representing 1-amino-cyclopropane-1-carboxylic acid (ACC-) synthase gene, involved in ethylene biosynthesis, was also mapped. As in previous mapping studies for watermelon, a large number of AFLP and SRAP markers were skewed away from the 1:1 segregation ratio, and had to be excluded from the final mapping analysis. The stringent mapping criteria (JoinMap 3.0 mapping program) produced linkage groups with marker order consistent with those reported in previous mapping study for watermelon.
\end{abstract}

Cultivated watermelon accounts for $2 \%$ of the world area devoted to vegetable crops [Food and Agricultural Organization of the United Nations (FAO), 1995]. In the United States, watermelon production has increased from 1.09 million $\mathrm{Mg}$ in 1980 to 3.54 million $\mathrm{Mg}$ in 2003, with a farm value of $\$ 310$ million [U.S. Department of Agriculture (USDA), 2003]. The traditional watermelon cultivars are diploids $(n=11$; Shimotsuma, 1963). However, in recent years, there has been an increased demand by consumers for seedless (triploid) watermelons, and production has increased considerably. During 2003, over $60 \%$ of the watermelons produced in the United States were seedless (USDA, 2003). There is a continuous need to enhance disease and pest resistance in watermelon cultivars, improve fruit quality, and develop new seedless watermelon cultivars suitable to consumer

\footnotetext{
Received for publication 11 Oct. 2005. Accepted for publication 15 Dec. 2005. The authors gratefully acknowledge Laura Pence for her technical work in constructing the map. Use of trade names does not imply endorsement of the products names nor criticism of similar ones not named.

'Corresponding author: alevi@saa.ars.usda.gov
}

needs. Molecular markers are useful in identifying and isolating genes controlling disease resistance, or genes controlling fruit quality (Cobos et al., 2005; Liebhard et al., 2003; Mokrani et al., 2002; Soriano et al., 2005). As in other crop plants, including coffee (Coffea arabica L.) (Herrera et al., 2002) and banana (Musa L.) (Nwakanma et al., 2003), molecular markers can be useful in identifying and in selecting for triploid hybrid plants of watermelon.

High-density genetic maps are being integrated in breeding programs of many crop plants. They are also useful in positional cloning of genes and in elucidating the genetic mode of complex traits that do not display Mendelian segregation (Lee, 1995). Cucumis sativus L. (cucumber) and Cucumis melo L. (melon) received the greatest attention among cucurbit species with respect to genome mapping (Fazio et al., 2003; Silberstein et al., 2003). Linkage mapping of watermelon genome has been lagging behind the maps constructed for these species. A few linkage maps were constructed for watermelon in recent years. However, these maps are based on isozymes (Navot and Zamir, 1987; Zamir et al., 1984) or RAPD markers (Hashizume et al., 1996, 2003; Hawkins et al., 2001; Levi et al., 2001c, 2002), and 
do not cover all regions of watermelon genome. Many markers (including SRAP, SSR, and AFLP markers) are still required for constructing a dense map that can be used effectively in watermelon breeding programs, and in isolating genes that control fruit quality or confer resistance to diseases and pests. Most of the watermelon cultivars developed in North America during the last two centuries share a narrow genetic base (Levi et al., 2001a, 2001b). Due to the low DNA polymorphism among watermelon cultivars, most of the maps constructed so far for watermelon used genetic mapping populations derived from a wide cross between a watermelon cultivar ( $C$. lanatus var. lanatus) and a U.S. plant introduction (PI) of the related subspecies $C$. lanatus var. citroides (Zhang et al., 2004).

Hawkins et al. (2001) and Levi et al. (2004b) attempted to construct a linkage map for watermelon using $\mathrm{F}_{2}$ or $\mathrm{F}_{3}$ populations derived from a wide cross between PI 296341 (C. lanatus var. citroides) and the watermelon cultivar New Hampshire Midget (NHM). However, most of the markers in these populations were skewed away from the expected segregation ratio (3:1) and could not be mapped with high confidence. In an attempt to reduce the number of skewed markers we used a testcross population [(Griffin $14113 \times$ NHM) x PI 386015] where the testcross parent (PI 386015) is distant from both parents of the $F_{1}$ plant (Griffin 14113 and NHM) (Levi et al., 2002). This testcross population was successfully used in our initial mapping of watermelon using RAPD and ISSR markers (Levi et al., 2002). Our objective is to extend the initial map using different types of DNA markers, and in a later stage isolate genes controlling disease and pest resistances and genes controlling watermelon fruit qualities. SRAP, AFLP, and SSR markers have been used in constructing maps for various crops (Li et al., 2003), but not for watermelon. It is vital to experiment with these marker types, determine their polymorphism, segregation, and distribution on watermelon genome, and further explore the linkage regions they cover.

This paper reports the construction of an extended genetic linkage map for watermelon using a testcross population [(Griffin $14113 \times$ NHM) x PI 386015] and examines the efficiency of SRAP, AFLP, and SSR markers vs. ISSR and RAPD markers in constructing the map.

\section{Materials and Methods}

Plant material. Parental plants that included Griffin 14113 (provided by R. Jarret, USDA Plant Genetic Resources Conservation Unit, Griffin, Ga.), NHM, their $\mathrm{F}_{1}$ hybrid plant, and the testcross parent plant (PI 386015) were grown in the greenhouse together with 98 plants of the testcross progeny [(Griffin $14113 \mathrm{x}$ NHM) x PI 386015]. Four weeks after germination young leaves $(10 \mathrm{~g})$ were collected from each plant, and stored at $-80^{\circ} \mathrm{C}$.

ISOLATION OF DNA. To avoid co-isolation of polysaccharides, polyphenols, and other secondary compounds that damage DNA, we used an improved procedure for isolation of DNA from young leaves of watermelon (Levi and Thomas, 1999).

AFLP PROCEDURE. The AFLP procedure developed by Vos et al. (1995) was modified using a commercially available kit (Plant Mapping Kit-RegularPlant Genome;Applied Biosystems, Foster City, Calif.). According to the manufacturer's protocol, a highquality genomic DNA sampled [500 ng; intact and with a 260/280 optical density (OD) ratio of 1.8] from each plant was used in the AFLP analysis with EcoRI-MseI, and PstI-MseI restriction enzymes and primer combinations (Table 1) as described by Levi et al. (2004a). Reactions were analyzed on a $6 \%$ acrylamide gel using a Perkin Elmer ABI-373 Sequencer (Applied Biosystems) as described by Levi et al. (2004a), or on a capillary system using the CEQ8800 DNA sequencer (Beckman Coulter, Fullerton, Calif.). For visualization of DNA fragments on the CEQ8800 the EcoRI or PstI selective primers were labeled with one of three WellRED dye labels (D2, D3, or D4; Proligo, Boulder, Colo.), as described below for the SRAP analysis.

SRAP ANALYSIS. The SRAP procedure is based on PCR amplification of open reading frames (ORFs), using a forward and reverse primers designed to preferentially amplify exon (rich in $\mathrm{C}$ and $\mathrm{G}$ nucleotides) and intron regions (rich in $\mathrm{A}$ and $\mathrm{T}$ nucleotides), respectively. The forward primer is a 14 nucleotide sequence rich in $\mathrm{C}$ and $\mathrm{G}$, and three selective bases at the $3^{\prime}$ end, while the reverse primer is a 15 nucleotide sequence rich in $\mathrm{A}$ and $\mathrm{T}$ and three selective bases at the $3^{\prime}$ end. The variation in exon, intron or promoter region sequences produces the polymorphism (Li and Quiros, 2001).

Twenty-four SRAP primer combinations were (Table 2) tested with the testcross parents (NHM, Griffin 114113, and PI 396015). For visualization with the CEQ8800 DNA sequencer, the forward primers were labeled with one of three WellRED dyes (D2, D3, or D4) (Proligo). To confirm correct dye balance, the amount of dye-labeled primer added to the PCR reaction was optimized for each primer. Each 10- $\mu \mathrm{L}$ PCR reaction contained $1 \mathrm{X}$ PCR buffer (Promega, Madison, Wis.), $2.5 \mathrm{~mm} \mathrm{MgCl}_{2}, 200 \mu \mathrm{MdNTPs}$ (Sigma, St. Louis), $30 \mathrm{ng}$ of reverse primer (IDT, Coraville, Iowa), 0.4 units Taq DNA polymerase (Promega) and $25 \mathrm{ng}$ of DNA. In addition, WellRED-dye-labeled forward primers were added in the following optimized amounts: $15 \mathrm{ng}$ for a D4 primer; $30 \mathrm{ng}$ for a D3 primer; or $75 \mathrm{ng}$ for a D2 primer. Samples were subjected to the following thermal profile for amplification in a PCR thermocycler (MJ): 5 min of DNA denaturizing at $94{ }^{\circ} \mathrm{C}$, five cycles of three steps: 1 min of denaturing at $94^{\circ} \mathrm{C}, 1 \mathrm{~min}$ of annealing at $35^{\circ} \mathrm{C}$ and $2 \mathrm{~min}$ of elongation at $72{ }^{\circ} \mathrm{C}$. In the following 30 cycles the annealing temperature was increased to $50{ }^{\circ} \mathrm{C}$, with a final elongation step of $5 \mathrm{~min}$ at $72{ }^{\circ} \mathrm{C}$ ( $\mathrm{Li}$ and Quiros, 2001). The PCR products were analyzed using the CEQ8800 capillary sequencer (Beckman Coulter) by pooling $0.3 \mu \mathrm{L}$ of the D4 reaction, $0.3 \mu \mathrm{L}$ of the $\mathrm{D} 3$ reaction, $0.5 \mu \mathrm{L}$ of the $\mathrm{D} 2$ reaction, 0.28 $\mu \mathrm{L}$ of D1 size standard, and $30 \mu \mathrm{L}$ of deionized formamide in a 96-well PCR plate. Samples were run on the CEQ8800 capillary sequencer using the fragment 3 parameters and analyzed using the built-in fragment analysis software provided with the CEQ8800 system. Fragments that ranged in size of 75-400 bp could be scored with high confidence.

SSR ANALYSIS. Microsatellite sequences were isolated from a genomic DNA of the watermelon cultivar Dixielee according to the procedure used by Reddy et al. (2001) for developing cotton SSRs. Following sequencing, primer pairs were designed for these DNA fragments containing SSR motifs (Table 4). The primer pairs were first tested with the testcross parents (Griffin 14113, NHM, and PI386015). PCR was performed in $10-\mu \mathrm{L}$ reactions containing $1 \mathrm{X}$ PCR buffer (Promega), $2.5 \mathrm{~mm} \mathrm{MgCl}_{2}$, $100 \mu \mathrm{M}$ dNTPs (Sigma), $0.25 \mu \mathrm{M}$ of each primer pair (Sigma Genosys, Houston), 0.2 units Taq DNA polymerase (Promega), and $25 \mathrm{ng}$ of DNA. The PCR conditions were identical to that of Saal et al. (2001), and annealing temperatures ranged from 49 to $68{ }^{\circ} \mathrm{C}$ (Table 1 ). The PCR products tested first with the parental lines were separated on a $6 \%$ native polyacrylamide gel with ethidium-bromide in the running buffer and visualized on an ultraviolet (UV) transilluminator (Saal et al., 2001). The SSR markers that were polymorphic between the parental lines were 
Table 1. The AFLP primer pairs, their designation, and the size of markers that were suitable for mapping experiments with the testcross population used for constructing a linkage map for watermelon ( $\mathrm{E}=\mathrm{EcoRI}, \mathrm{M}=\mathrm{Mse}, \mathrm{P}=\mathrm{Pst}$ ).

\begin{tabular}{|c|c|c|}
\hline Primer pair & Designation & Markers size $(b p)$ \\
\hline EAAC - MCAG & EACAG & $89 \mathrm{c}, 99 \mathrm{c}^{\mathrm{z}}, 101^{\mathrm{z}}, 163 \mathrm{c}, 219 \mathrm{c}^{\mathrm{z}}, 248,312 \mathrm{c}^{\mathrm{z}}, 369 \mathrm{c}, 373 \mathrm{c}^{\mathrm{z}}, 425$ \\
\hline EACA - MCAC & ECAAC & $57 \mathrm{c}, 91 \mathrm{c}^{\mathrm{z}}, 95$ \\
\hline EACA - MCTG & ECATG & $90,139 \mathrm{c}, 174 \mathrm{c}^{\mathrm{z}}, 176 \mathrm{c}, 193^{\mathrm{z}}, 195^{\mathrm{z}}, 223 \mathrm{c}$ \\
\hline EACC - MCAA & ECCAA & $77 \mathrm{c}, 125,162,220 \mathrm{c}, 238 \mathrm{c}^{\mathrm{z}}, 482 \mathrm{c}^{\mathrm{z}}$ \\
\hline EACC - MCAC & ECCAC & $98 \mathrm{c}, 112 \mathrm{c}^{\mathrm{z}}, 161 \mathrm{c}, 224 \mathrm{c}^{\mathrm{z}}, 257 \mathrm{c}^{\mathrm{z}}, 454 \mathrm{c}$ \\
\hline EACC - MCAT & ECCAT & $90,92 \mathrm{c}, 101 \mathrm{c}, 141 \mathrm{c}, 145 \mathrm{c}, 463 \mathrm{c}$ \\
\hline EACC - MCTG & ECCTG & $93 c^{z}, 310 c$ \\
\hline EAGC - MCAG & EGCAG & $94 \mathrm{c}, 129 \mathrm{c}, 180,184 \mathrm{c}, 215^{\mathrm{z}}, 244 \mathrm{c}^{\mathrm{z}}, 252 \mathrm{c}^{\mathrm{z}}$ \\
\hline EAGC - MCTA & EGCTA & $79 \mathrm{c}, 199,240,368 \mathrm{c}$ \\
\hline EAGG - MCTT & EGGTT & $93,98 \mathrm{c}, 188 \mathrm{c}^{\mathrm{z}}, 252 \mathrm{c}, 263 \mathrm{c}^{\mathrm{z}}, 382 \mathrm{c}^{\mathrm{z}}$ \\
\hline EAGG - MCTC & EGGTC & $\begin{array}{l}114 c^{z}, 117^{z}, 120 c, 122,151,171,186 \mathrm{c} \\
199 c^{z}, 223 c^{z}, 302 c, 308 c^{z}\end{array}$ \\
\hline EAAG - MCTG & EAGTG & $118,213^{\mathrm{z}}, 269 \mathrm{c}, 271,272 \mathrm{c}^{\mathrm{z}}, 408 \mathrm{c}^{\mathrm{z}}$ \\
\hline EAAG - MCTC & EAGTC & $84 c, 86,259,272 c, 492 c^{z}$ \\
\hline EAAG - MCAT & EAGAT & $\begin{array}{l}104 c^{z}, 113 c^{z}, 121 c^{z}, 123^{z}, 128 c, 135 c, 138 c^{z}, 152 c^{z}, \\
165 c^{z}, 171 c^{z}, 172^{z}, 197^{z}, 226,427 c^{z}, 455^{z}\end{array}$ \\
\hline EAGG - MCAG & EGGAG & $93 c, 117 c, 147 c^{z}, 202^{z}, 205 c, 226 c^{z}, 237 c, 329 c, 334 c^{z}$ \\
\hline EAGG - MCAA & EGGAA & $91 c^{z}, 111 c, 135 c, 157 c, 344 c$ \\
\hline EACC - MCTA & ECCTA & $90,138 \mathrm{c}, 187 \mathrm{c}^{\mathrm{z}}, 257 \mathrm{c}, 338 \mathrm{c}$ \\
\hline EACA - MCAA & ECAAA & $89 c, 100$ \\
\hline EAAG - MCAG & EAGAG & $\begin{array}{l}81 \mathrm{c}, 91,100 \mathrm{c}, 121 \mathrm{c}^{\mathrm{z}}, 122^{\mathrm{z}}, 125 \mathrm{c}, 126^{\mathrm{z}}, 130 \mathrm{c}, 160 \mathrm{c}^{\mathrm{z}}, 174 \mathrm{c}^{\mathrm{z}}, \\
175^{\mathrm{z}}, 181 \mathrm{c}, 209 \mathrm{c}, 219 \mathrm{c}, 226 \mathrm{c}, 238 \mathrm{c}, 251 \mathrm{c}, 259 \mathrm{c}, 265^{\mathrm{z}}, \\
272,280 \mathrm{c}^{\mathrm{z}}, 288 \mathrm{c}, 292 \mathrm{c}^{\mathrm{z}}, 330 \mathrm{c}^{\mathrm{z}}, 332 \mathrm{c}^{\mathrm{z}}, 370 \mathrm{c}, 453 \mathrm{c}\end{array}$ \\
\hline PACG - MCAT & PCGAT & $142^{\mathrm{z}}, 347$ \\
\hline $\mathrm{PACG}-\mathrm{MCCT}$ & PCGCT & $82 c^{z}, 89 c^{z}, 106,117 c^{z}, 121 c, 178^{z}$ \\
\hline PACT - MCAA & PCTAA & 93 \\
\hline PACT - MCCG & PCTCG & $102 \mathrm{c}, 151,153,175 \mathrm{c}, 217 \mathrm{c}, 223 \mathrm{c}, 301 \mathrm{c}$ \\
\hline PACT - MCGA & PCTGA & $120,176^{\mathrm{z}}$ \\
\hline PAGA - MCAA & PGAAA & $128 \mathrm{c}, 159^{\mathrm{z}}, 183^{\mathrm{z}}, 129$ \\
\hline PAGA - MCGA & PGAGA & $239 \mathrm{c}, 241^{\mathrm{z}}$ \\
\hline PAGG - MCCC & PGGCC & $82,95,104,131,135 c, 206 \mathrm{c}, 231$ \\
\hline PAGG - MCCT & PGGCT & $81 c, 89 c, 393$ \\
\hline PATA - MCCC & PTACC & $94 c, 137 \mathrm{c}$ \\
\hline PATA - MCGT & PTAGT & $138 \mathrm{c}, 159^{\mathrm{z}}, 183^{\mathrm{z}}$ \\
\hline PAAG - MCAT & PAGAT & 181 \\
\hline PACA - MCAA & PCAAA & 114 \\
\hline PACT - MCAC & PCTAC & $288^{\mathrm{z}}$ \\
\hline PACT - MCCA & PCTCA & $151 \mathrm{c}$ \\
\hline PACT - MCCC & PCTCC & $113 \mathrm{c}, 114 \mathrm{c}, 130 \mathrm{c}, 179,180$ \\
\hline PACT - MCCT & PCTCT & $100 \mathrm{c}, 110 \mathrm{c}, 132,148 \mathrm{c}^{\mathrm{z}}, 152$ \\
\hline PATA - MCAA & PTAAA & $173 \mathrm{c}$ \\
\hline PATA - MCGA & PTAGA & $168 c, 296 c^{z}$ \\
\hline PATA - MCGC & PTAGC & $114 \mathrm{c}, 130 \mathrm{c}, 180$ \\
\hline PATA - MCGT & PTAGT & $140 \mathrm{c}, 212,413 \mathrm{c}$ \\
\hline
\end{tabular}

${ }_{2}$ Markers that could be ordered on the linkage map.

further analyzed for mapping with the testcross population using the CEQ8800 capillary sequencer. For visualization of fragments on the CEQ8800 DNA sequencer the SSR forward primers were labeled with one of three WellRED dye labels (D2, D3, or D4; Proligo), as described above for the SRAP analysis.

RAPD AND ISSR PROCEDURES. The RAPD and ISSR marker analyses were performed as described in previous studies (Levi et al., 1993, 2001c, 2002).

MARKER SCORING. Markers that were unique to one of the parents, Griffin 14113 or NHM, and were present in the $\mathrm{F}_{1}$ parent, but missing in the testcross parent (PI 386015), could be scored for mapping in the testcross population (Fig. 1). The AFLP markers were scored based on their presence or absence using Genescan/ Genotyper software (Applied Biosystems) for AFLP fragments, or the using the built-in fragment analysis software (provided with the Beckman CEQ8800 system) for SRAP and SSR fragments.

LINKAGE ANALYSIS OF MARKERS IN THE TESTCROSS POPULATION. Prior to linkage analysis segregation rates were scored for each marker. Segregation ratio distortion was evaluated with a chi-square test for goodness of fit based on the segregation expectations of 1:1 for markers in a testcross or $\mathrm{BC} 1$ population (Van Ooijen and Vooripps, 2001), and skewed markers were excluded from the analysis (Table 5). Linkage analysis was performed with the JoinMap version 3.0 software (Van Ooijen and Vooripps, 2001) with default options. All markers were analyzed for linkage, and recombination fractions were converted into map distances (centimorgans) with the Kosambi function. LOD scores of 2.0-6.0 were used for grouping of markers, followed by high threshold LOD scores of 7.0-10.0 for final mapping of markers in each linkage group. Loci showing weak or suspect linkages were removed from the analysis (Van Ooijen and Vooripps, 2001).

Marker nomenclature. The AFLP markers were designated by the last two nucleotides on the $3^{\prime}$ of the EcoRI and MseI primers, respectively. For example marker EAGAG-122 represents the AFLP marker (with a size of $122 \mathrm{bp}$ ) produced by the selective primers EcoRI-AAG and MseI-CAG. The RAPD or ISSR markers were designated by their serial number and their size. For example, the 500-bp marker produced by primer AW-07 (Operon Biotechnology, Alameda, Calif.), which is unique to Griffin 14113, was designated as AW07-500, and the 250-bp marker produced by primer No. 101 (Univ. of British Columbia, Vancouver), which is unique to NHM, was designated as 101-250c. The SRAP markers were designated by combining the forward and reverse primers (Table 2) with the marker size (Table 3). The SSRs provided by Seminis (Oxnard, Calif) are these beginning with $\mathrm{Cgb}$ or CLG, while the SSRs prepared at Alcorn State Univ. (Lorman, Miss.) are beginning with ASUW (Table 4). The RAPD or ISSR markers were designated by their serial number and their size. For example, the 500-bp marker produced by primer AW-07 (Operon Biotechnology), which is unique to Griffin 14113, was designated as AW07500; and the 250-bp marker produced by primerNo. 101 (Univ. of British Columbia), which is unique to NHM, was designated as U101-250c (Fig. 1; Levi et al., 2002).

\section{Results and Discussion}

POLYMORPHISM AND MAPPING EFFICIENCY OF MARKERS. The different marker types used in this study (RAPD, ISSR, AFLP, SRAP, and SSR markers) complement each other in the assembly of an extend linkage map. In a previous mapping study (Levi et al., 2002) 168 RAPD and 37 ISSR markers were used for mapping analysis. The AFLP, SRAP, and SSR marker data in this study were combined with these of the RAPD and ISSR markers to produce an extended linkage map (Table 5; Fig. 1). The AFLP, SRAP, or SSR markers were useful in covering linkage regions 
Table 2. SRAP forward primers that were labeled with a DNA sequencing dye (FLP) and the reverse unlabeled primers (RUP) used in different combinations to produce the SRAP markers (in Table 3) in watermelon.

\begin{tabular}{ll}
\hline FLP & Primer sequence 5 $^{\prime}$ to 3 $^{\prime}$ \\
\hline me1 & TGAGTCCAAACCGGATA \\
me2 & TGAGTCCAAACCGGAGC \\
me3 & TGAGTCCAAACCGGAAT \\
me4 & TGAGTCCAAACCGGACC \\
me5 & TGAGTCCAAACCGGAAG \\
ab1 & AGTGATTCAACCGGAGA \\
ab2 & AGTGATTCAACCGGATA \\
ab3 & AGTGATTCAACCGGAGC \\
RUP & Primer sequence 5' to 3' \\
ba1 & GTCGAGCTGCCAATTATA \\
ba2 & GTCGAGCTGCCAATTAAT \\
ba3 & GTCGAGCTGCCAATTTGC \\
ba4 & GTCGAGCTGCCAATTTTT \\
ba5 & GTCGAGCTGCCAATTAAA \\
ba6 & GTCGAGCTGCCAATTAAC \\
ba7 & GTCGAGCTGCCAATTAAG \\
ba8 & GTCGAGCTGCCAATTATT \\
ba9 & GTCGAGCTGCCAATTATC \\
ba10 & GTCGAGCTGCCAATTATG \\
ba11 & GTCGAGCTGCCAATTTGA \\
ba12 & GTCGAGCTGCCAATTTGT \\
em1 & GACTGCGTACGAATTAAT \\
em2 & GACTGCGTACGAATTTGC \\
em3 & GACTGCGTACGAATTGAC \\
em4 & GACTGCGTACGAATTTGA \\
em5 & GACTGCGTACGAATTAAC \\
em6 & GACTGCGTACGAATTGCA \\
\hline &
\end{tabular}

that were not readily covered by the RAPD or ISSR markers, and in merging small (RAPD-based) linkage groups (Levi et al., 2002) into larger linkage groups (as shown with linkage groups I, II, III, V, XI, and XV; Table 5; Fig. 1).

POLYMORPHISM AND SCREENING OF AFLP MARKERS. Although AFLP markers (MseI-EcoRI) are highly polymorphic among watermelon cultivars (Levi et al., 2004a), a relatively small number of these markers (142 of 707 markers; 20.1\%) were suitable for the mapping experiment with the testcross population. Nineteen AFLP primer pairs (MseI-EcoRI) were tested against the parents Griffin 14113 and NHM, their $\mathrm{F}_{1}$ hybrid, and the testcross parent PI 386015. Each AFLP primer pair produced $\approx 12$ to 48 markers (75-450 bp). However, only a few of these markers (2-7) were suitable for the mapping experiment with the testcross population (Table 1). These AFLP markers were uniquely present in one of the donor parents (Griffin 14113 or NHM) and in the $\mathrm{F}_{1}$ hybrid, but absent in the testcross parent PI 386015. A total of 142 AFLP markers (38 markers unique to Griffin 14113 and 104 markers unique to NHM) were suitable for the mapping analysis using the testcross population (Table 1). However, 76 (54.9\%) of these markers were skewed from the expected 1:1 segregation ratio and had to be excluded from the final linkage analysis. Sixty-six AFLP markers were used in the final linkage analysis. Of these, 58 could be mapped with high confidence on the linkage map (Tables 1 and 5; Fig. 1). Of the 58 AFLP markers that could be mapped, 24 markers were clustered on linkage group XII and eight markers were on linkage group X. The other 26 AFLP markers are mapped on different linkage regions (Table 5; Fig. 1). Clustering of AFLP markers was reported in different mapping studies of crop plants as shown with tomato (Lycopersicon esculentum Mill.) (Saliba-Colombani et al., 2000) and melon (Cucumis melo L.) genomes (Perin et al., 2002).

As compared with the MseI-EcoRI primer pairs, the $21 P s t \mathrm{I}-$ $M s e$ IAFLP primer pairs tested in this study produced a low number (62) of polymorphic markers suitable for the mapping experiment with the testcross population (Table 1). Of these 62 markers, 42 $(67.7 \%)$ were skewed from the expected 1:1 segregation ratio and had to be excluded from the mapping analysis (Table 1). Of the 20 markers used in the final mapping analysis, 13 could be mapped with high confidence (Tables 1 and 5; Fig. 1). Three of the PstI-MseI markers were clustered on linkage group XIX, while the 10 other markers were mapped on different linkage groups (Table 5; Fig. 1).

POLYMORPHISM AND SCREENING OF SRAP MARKERS. The SRAP markers proved to be efficient and reliable in the mapping analysis in this study. Each of the 69 primer pairs tested (Table 2) produced $\approx 12-28$ DNA fragments. About $1-7$ of these fragments were suitable for the mapping experiment with the testcross population (total of 223 fragments produced by 69 SRAP primer pairs) (Table 3). However, 120 of the 223 markers were skewed from the expected 1:1 segregation ratio, and had to be excluded from the final mapping analysis (Tables 3 and 5). Of the remaining 103 SRAP markers, 93 could be mapped with high confidence in the final mapping analysis (Table 3 and 5; Fig. 1). In contrast with the AFLP markers, most of the SRAP markers did not cluster and are distributed on most linkage groups (Table 5; Fig. 1).

POLYMORPHISM AND SCREENING OF SSR MARKERS. Forty-three SSR primer pairs were tested for polymorphism between the testcross parents. Of these, 11 primer pairs produced polymorphic markers (total of 20 markers) suitable for the mapping experiment. Two of these markers had distorted segregation, and were excluded from the mapping analysis. Fourteen of the remaining 18 markers, could be ordered on the linkage map (Tables 4 and 5; Fig. 1). Four markers (CLG7996-158, CLG8020-357c, CLG8020-337, and ASUW2-165) are on linkage group V, three (ASUW13-128, ASUW13-105, CLG7992-112) are on linkage group XVII, two (ASWU19-136, and ASUW19-121) are on linkage group VII, and two (CLG8218-281, CLG8218-297) are on linkage group IX. Markers Cgb4765-186, Cgb4767-179, and CLG7996-160 are on linkage groups II, XIII, and XIV, respectively (Table 5; Fig. 1).

MaPPING OF ETHYLENE BIOSYNTHESIS GENE. Trebitsh etal.(1997) have shown that in cucumber the Female locus $(F)$ is tightly linked to the 1-amino-cyclopropane-1-carboxylic acid (ACC) synthase (CS-ACSIG), a key regulatory enzyme in the ethylene biosynthetic pathway. Genomic DNA clones representing four distinct ACC-synthase genes in watermelon were isolated and sequenced (A. Salman and T. Trebitsh, unpublished data). These clones were designated as Cit-ACS1 (1342 bp), Cit-ACS2 (892 bp), Cit-ACS3 (1584 bp), and as Cit-ACS4 (914 bp). Different primer combinations were designed for each of these clones, and were used in PCR amplification experiments with the mapping parents. However, only clone Cit-ACS3 (designated in the map as ACS3-345) could be mapped with high confidence on the linkage map (Table 5; Linkage Group XVII; Fig. 1). The forward and reverse primers $\left(5^{\prime}\right.$-GCTAGAGTTTCGAAATGTACTAT and 5'-ACCAATTATTATTAC AATGGTG) produced a 345-bp fragment unique to Griffin 14113, which could be used for map- 
Table 3. SRAP primer pairs and the size of their markers used for mapping experiments with a watermelon testcross population.

\begin{tabular}{|c|c|c|c|}
\hline Primer pair & Marker size (bp) & Primer pair & Marker size (bp) \\
\hline Me1ba1 & $88 c, 136 c, 165 c, 185 c^{z}, 240 c^{z}, 269 c^{z}$ & Ab1ba4 & $77 c, 95 c$ \\
\hline Me1ba11 & $76 c^{z}, 184 c^{z}, 214,250^{z}$ & Ab1ba6 & $57,145 c, 159 c, 211,342$ \\
\hline Me1ba2 & $116,316 c^{z}, 330^{z}$ & Ab1ba7 & $71^{z}, 76,93,121,206 c^{z}$ \\
\hline Me1ba4 & $62 c^{z}, 79 c, 308 c, 402 c$ & Ab1ba8 & $64^{z}$ \\
\hline Me1ba5 & $174^{z}$ & Ab1ba9 & $92^{z}$ \\
\hline Me1em1 & $67 c^{z}, 175,196 c^{z}$ & Ab1em4 & $155 c^{z}$ \\
\hline Me1em3 & $103 c, 151 c, 281 c^{z}$ & Ab2ba1 & $90 c, 248 c, 421 c$ \\
\hline Me1em5 & $90 c, 102,263 c$ & Ab2ba4 & $68 c, 69,166 c, 282^{z}$ \\
\hline Me2ba1 & $352 c$ & Ab2ba6 & $60,63 c, 175 c, 204^{z}, 230^{z}, 356 c$ \\
\hline Me2ba3 & $110 c^{z}, 178 c^{z}, 376$ & Ab2ba7 & $76,117 \mathrm{c}$ \\
\hline Me2ba4 & $60 \mathrm{c}, 172^{\mathrm{z}}, 229^{\mathrm{z}}, 347$ & Ab2em1 & $89 c$ \\
\hline Me2ba5 & $88,172 c, 179^{z}, 226 c^{z}$ & Ab2em3 & $358 c, 369$ \\
\hline Me2em3 & $67 c, 68,126^{z}$ & Ab2em4 & $90 c^{z}$ \\
\hline Me2em4 & $74 c^{z}, 88 c, 113 c^{z}, 121 c, 136 c, 168 c^{z}$ & Ab2em5 & $114,126 c^{z}, 219,222 c^{z}, 247 c^{z}, 275,360 c^{z}$ \\
\hline Me2em5 & $64,182 c^{z}, 203,267 c^{z}$ & Ab2em6 & $92,110 c^{z}, 152,189 c^{z}$ \\
\hline Me2em6 & $60,147 c^{z}, 230 c, 297^{z}$ & Ab3ba11 & $159,213 c, 234$ \\
\hline Me3ba1 & $63 c^{z}, 83 c^{z}, 129,173 c^{z}, 383 c$ & Ab3em3 & $67,74 c^{z}, 208 c$ \\
\hline Me3ba10 & $80 c, 101 c, 320 c^{z}$ & Ab3em5 & $203^{z}, 235^{z}, 422$ \\
\hline Me3ba6 & $181 c^{z}, 187,286 c$ & Ab3em6 & $146 c^{z}, 148 c^{z}, 195^{z}$ \\
\hline Me3ba8 & 104 & Me1ba7 & $93 c^{z}, 116 c, 117 c$ \\
\hline Me3ba9 & $71,112 c^{z}$ & Me1ba8 & $192 c^{z}, 308 c^{z}, 402 c$ \\
\hline Me3em1 & $57,76 c^{z}, 129 c^{z}$ & Me1em6 & $154,401 c$ \\
\hline Me3em2 & $91^{z}$ & Me2ba10 & $81 c^{z}, 114 c^{z}$ \\
\hline Me4ba10 & $57 c, 68 c, 179^{z}, 234^{z}$ & Me2ba2 & $330 c$ \\
\hline Me4ba2 & $58 c, 64 c, 173 c^{z}, 291 c^{z}$ & Me2ba8 & $123 c, 127 c^{z}, 133 c^{z}, 147 c, 148 c^{z}, 147 c^{z}$ \\
\hline Me4ba3 & $79,99 c^{2}$ & Me2ba9 & 83,96 \\
\hline Me4ba4 & $166^{z}, 218 c, 222 c, 345 c^{z}$ & Me3ba11 & $86 \mathrm{c}, 170$ \\
\hline Me4ba5 & $57 c, 163 c^{z}, 179^{z}, 234^{z}$ & Me3ba12 & $310^{z}, 313^{z}$ \\
\hline Me4ba8 & $58 c, 82^{z}, 97 c^{z}$ & Me3ba5 & $57,74 c, 83,171 c z, 239 c$ \\
\hline Me4em3 & $64^{z}, 76 c, 82 c, 260$ & Me5ba1 & 167 \\
\hline Me4em5 & $101,193,203,268^{z}, 359^{z}$ & Me5ba7 & $229,283,289 c$ \\
\hline Ab1ba1 & $68,93,141^{z}, 144 c^{z}, 146 c, 256 c^{z}$ & Me5em3 & $204 c$ \\
\hline Ab1ba10 & $59 c, 65,71,77 c^{z}, 78^{z}, 171 c, 179$ & Me5em5 & $142 c^{z}, 204$ \\
\hline Ab1ba11 & $88 c, 90 c, 242 c^{z}, 340$ & Me5em6 & $77 c, 137 c, 169^{z}, 259 c, 299 c^{z}, 301 c^{z}$ \\
\hline Ab1ba2 & $92,311 c, 432 c$ & & \\
\hline
\end{tabular}

zMarkers that could be ordered on the linkage map.

Table 4. SSR primer pairs that produced markers suitable for the initial mapping analysis of watermelon. Markers designated with "c" at the right of the marker size were derived from the parent 'New Hampshire Midget.' Other markers were derived from the parent Griffin 14113.

\begin{tabular}{|c|c|c|c|c|c|}
\hline $\begin{array}{l}\text { Forward } \\
\text { primer }\end{array}$ & Primer sequence & $\begin{array}{c}\text { Reverse } \\
\text { primer }\end{array}$ & Primer sequence & $\begin{array}{c}\text { SSR } \\
\text { motif }\end{array}$ & $\begin{array}{l}\text { Fragment } \\
\text { size (bp) }\end{array}$ \\
\hline $\mathrm{Cgb} 4765-\mathrm{F}$ & TTCTCTTCATTCCСCCAAAATC & $\mathrm{Cgb} 4765-\mathrm{R}$ & ACGGGTGAGGGAAAACGAG & (CTT)13 & $186^{2}$ \\
\hline CLG8020-F & GGCAAGGATGGAGAGGGTATC & CLG8020-R & GGCCCTATTCCCCATAAGAC & $(\mathrm{GA}) 7$ & $337^{\mathrm{z}} / 357 \mathrm{c}^{\mathrm{z}}$ \\
\hline CLG7996-F & ATACCCTGGGTGAGAGATAGC & CLG7996-R & CCTTCCTTCCCGATTTTC & $(\mathrm{GA}) 6$ & $158^{z} / 160^{z}$ \\
\hline CLG7992-F & CTAACGCAATTTGAATCACTCAAA & CLG7992-R & GGTAAAATGAAATCAATTGTGGAGA & $(\mathrm{AAT}) 11$ & $112^{2}$ \\
\hline $\mathrm{Cgb} 4767-\mathrm{F}$ & GAGAGGGAAAGAAAAGAGGAGAG & Cgb4767-R & AACGGAGGATGATGATTTGGTA & $(\mathrm{CTT}) 13$ & $169 \mathrm{c} / 179^{\mathrm{z}}$ \\
\hline ASUW2-F & GCTTCGTTGTTGCTGCCGTTG & ASUW2-R & GCATAAAATCACACTCAAAC & $(\mathrm{AAG}) 12$ & $165 c^{z} / 192$ \\
\hline ASUW13-F & CTAGAGAAACCCCATC & ASUW13-R & CTCCACTCACATACACAG & $(\mathrm{AAG}) 18$ & $105^{\mathrm{z}} / 128^{\mathrm{z}}$ \\
\hline ASUW19-F & GTGTGTTTTTGCGTGTG & ASUW19-R & GGGCAAATCCAATAATCCAG & $(\mathrm{AAG}) 26$ & $121^{z} / 136^{z}$ \\
\hline Cgb5009-F & CAGTGGCACCGTCATCTAAAG & Cgb5009-R & AGTGGGGGATTCTCTTCCTAAG & $(\mathrm{GA}) 18$ & 216 \\
\hline CLG8288-F & CCCAACTCAATATTTTCGG & CLG8288-R & TTTTTTAATTTCGGGCTGG & (GA) 20 & 306 \\
\hline CLG8218-F & GAATTTCAAAATGTAGTTGTGC & CLG8218-R & GTTTTTCCCCTTACATAATTTAT & $(\mathrm{AAT}) 10$ & $281^{\mathrm{z}} / 297^{\mathrm{z}}$ \\
\hline
\end{tabular}

${ }^{\mathrm{z}}$ Markers that could be ordered on the linkage map. 
Table 5. The number of polymorphic markers tested for segregation with the testcross population used for constructing a linkage map for watermelon, the number of skewed markers that were excluded from the mapping analysis, marker distribution on linkage groups, the average distance between markers on each linkage group (DBM), and the total genetic distance (TGD) covered by each linkage group.

\begin{tabular}{|c|c|c|c|c|c|c|c|c|c|c|}
\hline Group & RAPDSs & ISSRs & $\begin{array}{c}\text { AFLP } \\
\text { EcoRI+MseI }\end{array}$ & $\begin{array}{c}\text { AFLP } \\
\text { PstI+MseI }\end{array}$ & SSRs & SRAPs & ACS & Totam & DBM & TGD \\
\hline $\begin{array}{l}\text { Total } \\
\text { analyzed }\end{array}$ & 178 & 38 & 142 & 62 & 20 & 222 & 3 & 665 & $(\mathrm{cM})$ & $(\mathrm{cM})$ \\
\hline Excluded & 15 & 0 & 76 & 42 & 2 & 120 & 0 & 99 & & \\
\hline I & 19 & 5 & 3 & 3 & 0 & 10 & 0 & 40 & 6.8 & 264.7 \\
\hline II & 16 & 4 & 0 & 0 & 1 & 11 & 0 & 32 & 6.9 & 212.1 \\
\hline III & 21 & 7 & 8 & 1 & 0 & 5 & 0 & 42 & $5.2^{\prime}$ & 211.2 \\
\hline IV & 19 & 5 & 2 & 0 & 0 & 8 & 0 & 34 & 4.7 & 155.2 \\
\hline V & 15 & 0 & 2 & 0 & 4 & 13 & 0 & 34 & 4.1 & 133.5 \\
\hline VI & 0 & 0 & 0 & 0 & 0 & 3 & 0 & 3 & 6.9 & 13.7 \\
\hline VII & 0 & 0 & 1 & 1 & 2 & 0 & 0 & 4 & 6.7 & 20.01 \\
\hline VIII & 1 & 2 & 0 & 1 & 0 & 0 & 0 & 4 & 12.8 & 38.4 \\
\hline IX & 0 & 0 & 0 & 0 & 2 & 2 & 0 & 4 & 18.2 & 54.6 \\
\hline $\mathrm{X}$ & 0 & 0 & 7 & 0 & 0 & 0 & 0 & 7 & 9.9 & 59.3 \\
\hline XI & 11 & 0 & 0 & 2 & 0 & 10 & 0 & 23 & 4.8 & 105.2 \\
\hline XII & 0 & 0 & 24 & 0 & 0 & 0 & 0 & 24 & 4.3 & 98.3 \\
\hline XIII & 11 & 2 & 1 & 1 & 1 & 4 & 0 & 20 & 5.1 & 96.8 \\
\hline XIV & 14 & 4 & 4 & 0 & 1 & 8 & 0 & 31 & 3.1 & 93.9 \\
\hline$X V$ & 14 & 0 & 5 & 1 & 0 & 11 & 0 & 31 & 4.2 & 124.9 \\
\hline XVI & 5 & 1 & 0 & 0 & 0 & 3 & 0 & 9 & 11.1 & 88.9 \\
\hline XVII & 4 & 0 & 0 & 0 & 3 & 3 & 1 & 11 & 7.9 & 79.5 \\
\hline XVIII & 1 & 0 & 1 & 0 & 0 & 2 & 0 & 4 & $23.0^{\prime}$ & 69.1 \\
\hline XIX & 0 & 0 & 0 & 3 & 0 & 0 & 0 & 3 & 28.5 & 57.01 \\
\hline $\begin{array}{l}\text { Total } \\
\text { mapped }\end{array}$ & 151 & 30 & 58 & 13 & 14 & 93 & 1 & 360 & 5.8 & 1976 \\
\hline
\end{tabular}

ping Cit-ACS3. Sex expression is greatly affected by ethylene. However, different studies indicate that it may have a complex role in flowering of watermelon (Sugiyama et al., 1998; Trebitsh et al., 1997). Presently, experiments are being conducted to isolate and map genes controlling ethylene biosynthesis and transduction in watermelon (A. Salman and T. Trebitsh, unpublished data).

MARKER SEGREgation. In contrast with the RAPD, ISSR, and SSR markers, most of the AFLP (EcoRI-MseI; PstI-MseI) and SRAP markers tested in this study were skewed away $(P<0.05)$ from the expected 1:1 ratio among testcross progeny (Table 5) and had to be excluded from the mapping analysis. In our recent study with an $\mathrm{F}_{2}$ mapping population (PI 296341-FR x NHM) most of the AFLP markers (80\%) were skewed from the expected $3: 1$ ratio (Levi et al., 2004b). Similarly, $47.5 \%$ and $48.0 \%$ of markers (RAPDs) were skewed in $\mathrm{F}_{2}$ and $\mathrm{F}_{3}$ mapping populations derived from the same parental lines (PI 296341-FR and NHM) (Hawkins et. al., 2001). In an initial linkage map constructed for watermelon using $\mathrm{aBC}_{1}$ population [(PI296341 $\left.\mathrm{xNHM}\right) \times \mathrm{NHM}$ ] $25.7 \%$ of the RAPD markers were skewed from the expected segregation (Levi et al., 2001c). In a recent mapping study using recombinant inbred lines (RILs; derived from a cross between PI 296341 and the high quality Chinese inbred line 97103) 28\% of the mapping markers (RAPDs and ISSRs) were skewed away from the 3:1 segregation ratio $(P<0.05)$ (Zhang et al., 2004). Skewed segregation frequently occurs in populations derived from interspecific crosses and may be a result of differences in genes controlling the reproduction processes (Zamir and Tadmor, 1986), or due to a meiotic drive where a chromosome with unique structural features or genetic properties render selective advantage or disadvantage to its respective gametes or zygotes (Buckler et al., 1999; Cameron and Moav, 1957; Lyttle, 1991; Sandler and
Novitski, 1957), as first shown in maize (Zea mays L.) by Rhodes (1942). However, the possibility of amplifying bands of the same size which represent different gene loci (non-single copy loci) may also play a role in the skewed segregation of some of these markers, as has been shown in PCR-RAPD analysis for different plant species (Amarger and Mercier, 1995).

The large number of skewed AFLP and SRAP as compared with the RAPD, ISSR and SSR markers is likely to be due to distinct properties of the genomic regions covered by these marker types. Higher rate of skewed AFLP or RAPD markers versus RFLP markers were found in mapping population of different crops including tomato (Saliba-Colombani et al., 2000), rice (Oryza sativa L.) (Cho et al., 1998), and melon (Perin et al., 2002). In contrast, in barley (Hordeum vulgare L.), a larger number of RFLP markers were skewed than AFLP markers (Becker et al., 1995). Highly skewed markers may contribute to overestimation of recombination frequency and to loose linkages between markers. Conversely, they may cause the merging of two linkage groups (Saliba-Colombani et al., 2000). Thus, in this study, the skewed markers had to be excluded from the mapping analysis.

MaP CONSTRUCTION. A total of 653 markers (222 SRAP, 204 AFLP, 168 RAPD, 38 ISSR, and 21 SSR markers) were tested for mapping. Of these, 224 markers were skewed away from the expected 1:1 segregation ratio and had to be excluded from the linkage analysis. Four-hundred and twenty-nine markers were tested for linkage analysis. Of these, 36 could be ordered with high confidence on the linkage map (Table 5; Fig. 1). The map consists of 12 large linkage groups (119.6-373 cM), and 7 small to midsize linkage groups (47.9-91.4 cM) (Table 5; Fig. 1). Marker order is consistent with this on the linkage groups of the first map (Levi et al., 2002). However, in a few linkage groups the order of 


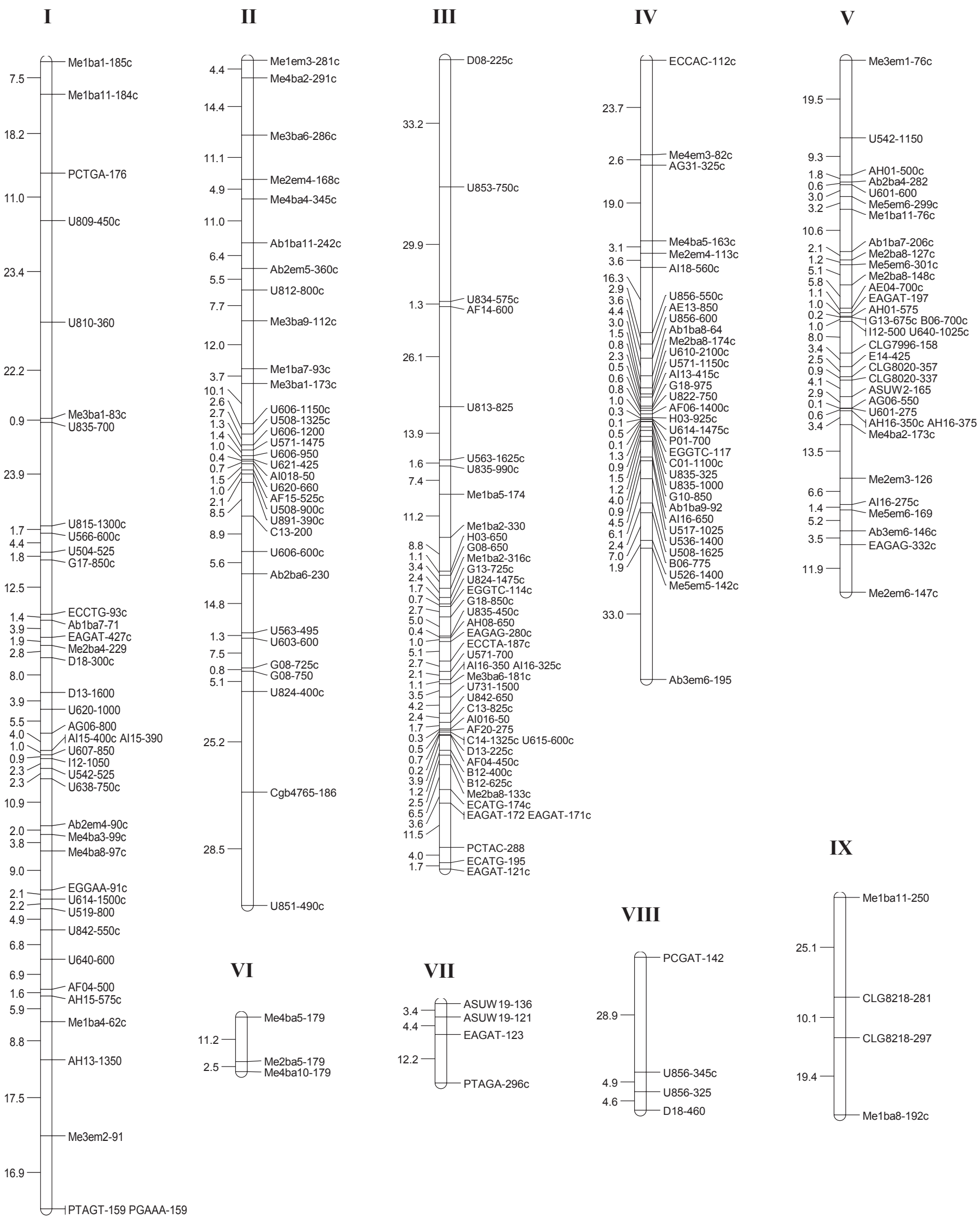

Fig. 1. An extended linkage map for watermelon. 
$\mathbf{X}$

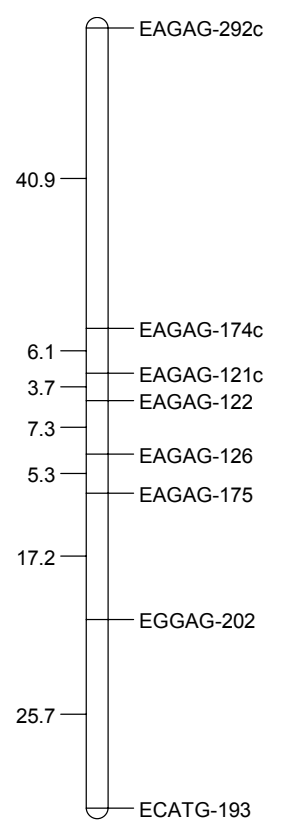

XI

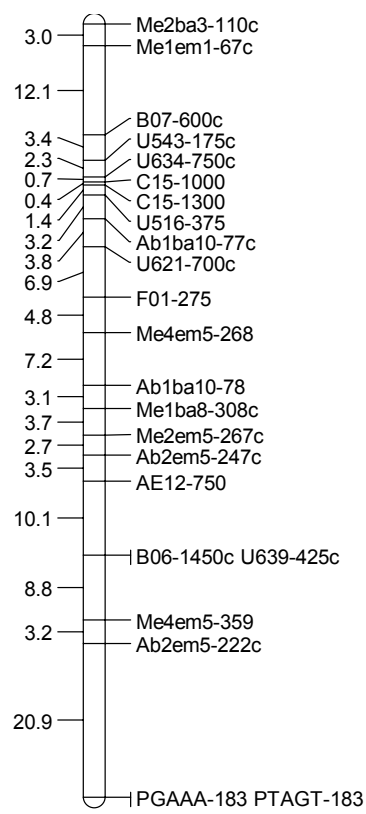

XII

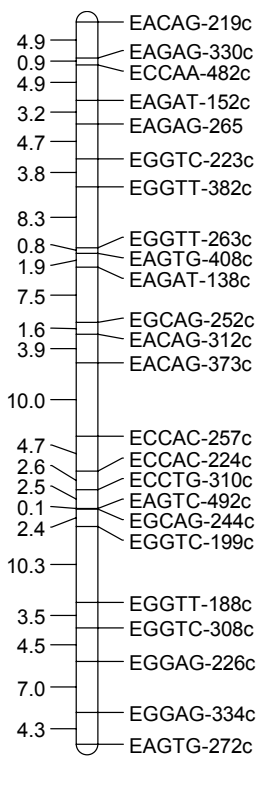

XIII

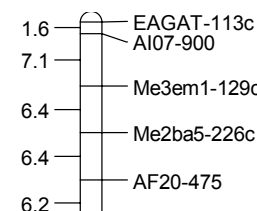

- U U $1571-650 \mathrm{c}$

$2.3 \longrightarrow \mathrm{AE} 12-575$

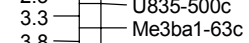

$1.6-\quad$ U - Me23-1025c

1.7- Me2em4-74c

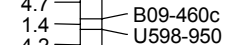

$2.2-\quad$ Al09-600
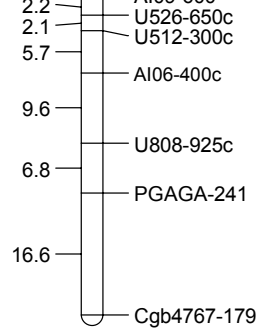

XIV

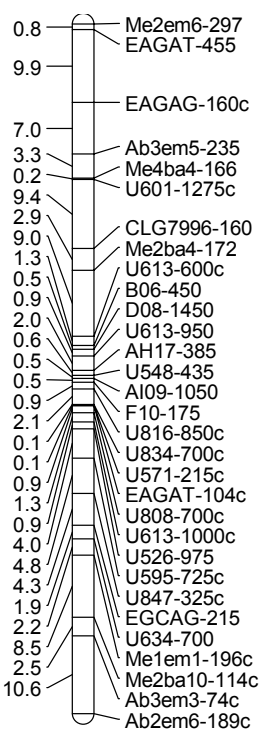

XV

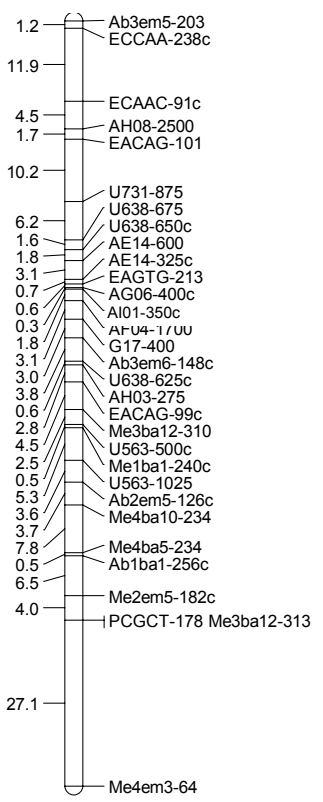

XVI

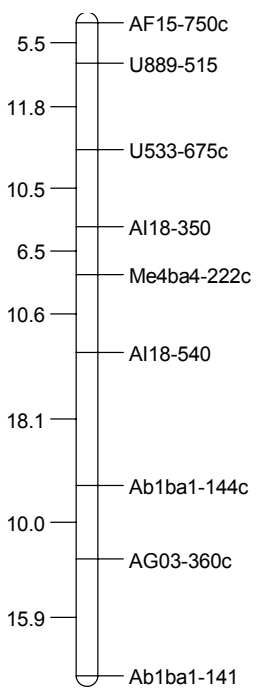

XVII

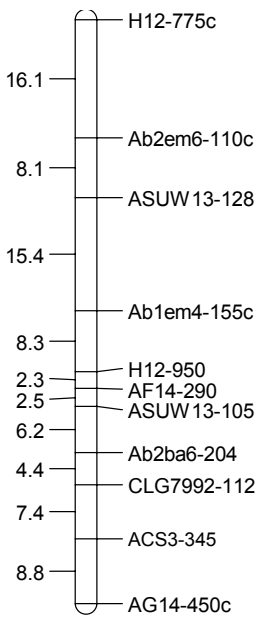

XVIII

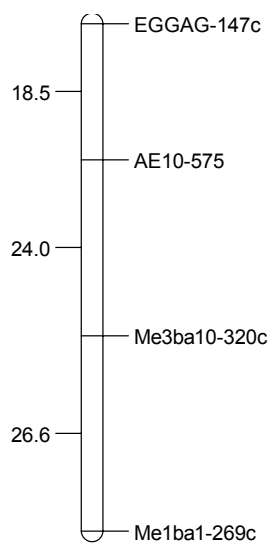

XIX

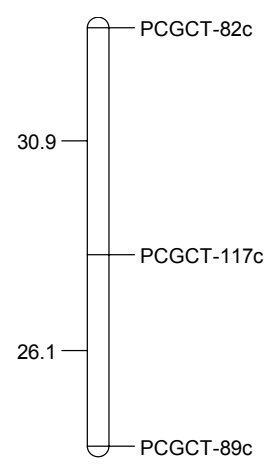

Fig. 1. Continued from previous page. An extended linkage map for watermelon.

RAPD or ISSR markers was slightly altered due to the integration of SRAP, AFLP, or SSR markers. A change in the order of a few RAPD markers was observed in linkage group XV (linkage group III in Levi et al., 2002) due to the integration of 6 AFLP and 11 SRAP markers into this group (Fig. 1). The marker order on linkage groups in this map is also consistent with marker order on linkage groups constructed for the $\mathrm{F}_{2}$ population (PI 296341 x NHM) (as shown by Levi et al., 2004b). A few markers in the present map (Fig. 1) were also detected in the study with $\mathrm{F}_{2} \mathrm{~S}_{7}$ RIL-based map constructed by Zhang et al. (2004). For example markers 835-325, P01-700, and G10-850 on linkage group V, and markers $824-1475 \mathrm{c}$ and $835-450 \mathrm{c}$ on linkage group III (Fig. 1) are in comparable genetic distances on linkage group I and II (respectively) on the map constructed by Zhang et al. (2004).

The stringent linkage analysis criteria used with the JoinMap analysis (Van Ooijen and Voorrips, 2001) resulted in a high 
number of linkage groups (Fig. 1). These criteria were used to avoid any possible mapping errors, making the map useful in merging experiments with other maps constructed for watermelon (Hashizume et al., 1996, 2003; Hawkins et al., 2001; Levi et al., 2001c; 2002; Zhang et al., 2004).

The total genetic distance of the first map constructed with the present population (141 RAPD and 27 ISSR markers) is 1166.2 with an average genetic distance of $8.1 \mathrm{cM}$ between markers (Levi et al., 2002). In this study, the addition of 93 SRAP, 71 AFLP, 11 RAPD, 3 ISSR, and 14 SSR markers increased the total genetic distance of the map to $1976 \mathrm{cM}$ with an average genetic distance of $5.8 \mathrm{cM}$ between markers.

Conclusions. Linkage analysis in watermelon is a challenging task because of low DNA polymorphism among watermelon cultivars, and on the other hand, the skewed segregation for large number of markers in mapping populations derived from crosses between PIs of $C$. lanatus var. citroides and watermelon cultivars (C. lanatus var. lanatus). The exclusion of a large number of skewed markers indicates that large regions of watermelon genome are still not mapped. Although AFLPs (MseI-EcoRI) produce a high number of polymorphic markers, a relatively small number of these markers was suitable for the mapping analysis. Most of the AFLP markers are ordered on linkage regions different from those discovered by RAPD, ISSR, and SSR markers. Although the large number of skewed SRAP markers, they appeared to be useful in the mapping analysis of watermelon genome. The SSR primers produced a relatively low number of markers useful for mapping. However, like the ISSR markers most of the SSR markers were not skewed from the 1:1 ratio and could be mapped. The large number of skewed AFLP and SRAP markers may reflect the non Mendelian segregation observed for different fruit and seed qualities in $\mathrm{F}_{2}$ populations derived from crosses between $C$. lanatus var. citroides accessions and watermelon cultivars (C. lanatus var. lanatus). A few markers in this map are also found in maps constructed for watermelon using an $\mathrm{F}_{2}$ population (Levi et al., 2004b) or a RIL population (Zhang et al., 2004). The order of these markers corresponds with those in the other maps (Levi et al., 2004b). The map presented in this study can be useful in merging the different maps constructed for watermelon (Hashizume et al., 2003; Hawkins et al., 2001; Zhang et al., 2004) and in mapping of markers linked to disease resistance or to fruit qualities.

\section{Literature Cited}

Amarger, V. and L. Mercier. 1995. Molecular analysis of RAPD DNA based markers: Their potential use for the detection of genetic variability in jojoba (Simmondsia chinensis L. Schneider). Biochimie 77:931-936.

Becker, J., P. Vos, M. Kuiper, F. Salamini, and M. Heun. 1995. Combined mapping of AFLP and RFLP markers in barley. Mol. Gen. Genet. 249:65-73.

Buckler, E.S., IV, T.L. Phelps-Durr, C.S. Buckler, R.K. Dawe, J.F. Doebley, and T.P. Holtsford. 1999. Meiotic drive of chromosomal knobs reshaped the maize genome. Genetics 153:415-426.

Cameron, D.R. and R. Moav. 1957. Inheritance in Nicotiana tabacum XXVII. Pollen Killer, an alien genetic locus inducing abortion of microspores not carrying it. Genetics 42:326-335.

Cho, Y.G., S.R. McCouch, M. Kuiper, M.R. Kang, J. Pot, J.T.M. Groenen, and M.Y. Eun. 1998. Integrated map of AFLP, SSLP and RFLP markers using a recombinant inbred population of rice (Oryza sativa L.). Theor. Appl. Genet. 97:370-380.

Cobos, M.J., M.J. Fernandez, J. Rubio, M. Kharrat, M.T. Moreno, J. Gil, and T. Millan. Alinkage map of chickpea (Cicerarietinum L.) based on populations from Kabuli $\times$ Desi crosses: Location of genes for resistance to fusarium wilt race 0. Theor. Appl. Genet. 110:1347-1353.

Fazio, G., J.E. Staub, and M.R. Stevens. 2003. Genetic mapping and QTL analysis of horticultural traits in cucumber (Cucumis sativus L.) using recombinant inbred lines. Theor. Appl. Genet. 107:864-874.

Food and Agricultural Organization of the United Nations. 1995. Production yearbook for 1994. No. 48. FAO, Rome.

Hashizume, T., I. Skimamoto, Y. Harushima, M. Yui, T. Sato, T. Imai, and M. Hirai. 1996. Construction of a linkage map for watermelon [Citrullus lanatus (Thunb.)Matsum \& Nakai] using random amplified polymorphic DNA (RAPD). Euphytica 90:265-273.

Hashizume, T., I. Shimamoto, and M. Hirai. 2003. Construction of a linkage map and QTL analysis of horticultural traits for watermelon [Citrullus lanatus (Thunb.) Matsum \& Nakai] using RAPD, RFLP and ISSR markers. Theor. Appl. Genet. 106:779-785.

Hawkins, L.K., F. Dane, T.L. Kubisiak, B.B. Rhodes, and R.L. Jarret. 2001. Linkage mapping in a watermelon population segregating for fusarium wilt resistance. J. Amer. Soc. Hort. Sci. 126:344-350.

Herrera, J.C., M.C. Combes, H. Cortina, G. Alvarado, and P. Lashermes. 2002. Gene introgression into Coffea arabica by way of triploid hybrids (C. arabica $\times$ C. canephora). Heredity 89:488-494.

Lee, M. 1995. DNA markers and plant breeding programs. Adv. Agron. 55:265-344.

Levi, A., L.J. Rowland, and J.S. Hartung. 1993. Production of reliable randomly amplified polymorphic DNA (RAPD) markers from DNA of woody plants. HortScience 28:1188-1190.

Levi, A. and C.E. Thomas. 1999. An improved procedure for isolation of high quality DNA from watermelon and melon leaves. Cucurbit Genetics Coop. Rpt. 22:41-42.

Levi, A., C.E. Thomas, A.P. Keinath, and T.C. Wehner. 2001a. Genetic diversity among watermelon (Citrullus lanatus and Citrullus colocynthis) accessions. Genet. Resources Crop Evol. 48:559-566.

Levi, A., C.E. Thomas, T.C. Wehner, and X. Zhang. 2001b. Low genetic diversity indicates the need to broaden the genetic base of cultivated watermelon. HortScience 36:1096-1101.

Levi, A., C.E. Thomas, X. Zhang, T. Joobeur, R.A. Dean, T.C. Wehner, and B.R. Carle. 2001c. A genetic linkage map for watermelon based on randomly amplified polymorphic DNA (RAPD) markers. J. Amer. Soc. Hort. Sci. 126:730-737.

Levi, A., C.E. Thomas, T. Joobeur, X. Zhang, and A. Davis. 2002. A genetic linkage map for watermelon derived from a testcross population: (Citrullus lanatus var. citroides $\mathrm{x} C$. lanatus var. lanatus) $\mathrm{x} C$. colocynthis. Theor. Appl. Genet. 105:555-563.

Levi, A., Thomas, C.E., Newman, M., Reddy, O.U.K., Zhang, X. and Y. Xu. 2004a. ISSR and AFLP markers sufficiently differentiated among American watermelon cultivars with limited genetic diversity. J. Amer. Soc. Hort. Sci. 129:553-558.

Levi A., C. E. Thomas, J. Thies, A. Simmons, Y. Xu, X. Zhang, O.U.K. Reddy, Y. Tadmor, N. Katzir, T. Trebitsh, S. King, A. Davis, J. Fauve, and T. Wehner. 2004b. Developing genetic linkage map for watermelon: Polymorphism, segregation and distribution of markers. Proc. 8th Eucarpia Meeting on Cucurbit Genetics and Breeding. p. 515-523.

Li, G., M. Gao, B. Yang, and C.F. Quiros. 2003. Gene for gene alignment between the Brassica and Arabidopsis genomes by direct transcriptome mapping. Theor. Appl. Genet. 107:168-180.

Li, G. and C.F. Quiros. 2001. Sequence-related amplified polymorphism (SRAP), a new marker system based on a simple PCR reaction: Its application to mapping and gene tagging in Brassica. Theor Appl Genet. 103:455-461.

Liebhard, R., B. Koller, L. Gianfranceschi, and C. Gessler. 2003. Creating a saturated reference map for the apple (Malus $\times$ domestica Borkh.) genome. Theor Appl Genet. 106:1497-1508.

Lyttle, T.W. 1991. Segregation distorters. Annu. Rev. Genet. 25:551557.

Mokrani, L., L. Gentzbittel, F. Azanza, L. Fitamant, G. Al-Chaarani, and A. Sarrafi. 2002. Mapping and analysis of quantitative trait loci for grain oil content and agronomic traits using AFLP and SSR in sunflower (Helianthus annuus L.). Theor. Appl. Genet. 106:149-156. 
Navot, N. and D. Zamir. 1987. Isozyme and seed protein phylogeny of the genus Citrullus (Cucrbitaceae). Plant Syst. Evol. 156:61-67.

Nwakanma, D.C., M. Pillay, B.E. Okoli, and A. Tenkouano. 2003. PCR-RFLP of the ribosomal DNA internal transcribed spacers (ITS) provides markers for the A and B genomes in Musa L. Theor. Appl. Genet. 108:154-159.

Perin, C., S. Hagen, V. De Conto, N. Katzir, Y. Danin-Poleg, V. Portnoy, S. Baudracco Arnas, J. Chadoeuf, C. Dogimont, and M. Pitrat. 2002. A reference map of Cucumis melo based on two recombinant inbred line populations. Theor. Appl. Genet., 104:1017-1034.

Reddy, O.U.K., A.E. Pepper, I. Abdurakhmonov, S. Saha, J.N. Jenkins, T. Brooks, Y. Bolek, and K.M. El-Zik. 2001. New Nucelotide and trinucleotide microsatellite marker resources for cotton genome research. J. Cotton Sci. 5:103-113.

Rhodes, M.M. 1942. Preferential segregation in maize. Genetics 27:395-407.

Saal, B., J. Plieske, J. Hu, C.F. Quiros, and D. Struss. 2001. Microsatellite markers for genome analysis in Brassica. II. Assignment of rapeseed microsatellites to the $\mathrm{A}$ and $\mathrm{C}$ genomes and genetic mapping in Brassica oleracea L. Theor. Appl. Genet. 102:695-699.

Sandler, L. and E.M. Novitski. 1957. Meiotic drive as an evolutionary force. Amer. Naturalist 91:105-110.

Saliba-Colombani, V., M. Causse, L. Gervais, and J. Philouze. 2000. Efficiency of RFLP, RAPD, and AFLP markers for the construction of an intraspecific map of the tomato genome. Genome 43:29-40.

Silberstein, L., I. Kovalski, Y. Brotman, C. Perin, C. Dogimont, M. Pitrat, J. Klingler, G. Thompson, V. Portnoy, N. Katzir, and R. Perl-Treves. 2003. Linkage map of Cucumis melo including phenotypic traits and sequence-characterized genes. Genome 46:761-73.
Soriano, J.M., S. Vilanova, C. Romero, G. Llacer, and M.L. Badenes. 2005. Characterization and mapping of NBS-LRR resistance gene analogs in apricot (Prunus armeniaca L.). Theor. Appl. Genet. 110:980-989.

Shimotsuma, M. 1963. Cytogenetic and evolutionary studies in the genus Citrullus. Seiken Jiho 15:24-34.

Sugiyama, K., T. Kanno, and M. Morishita. 1998. Evaluation method of female flower bearing ability in watermelon using silver thiosulfate (STS). J. Jpn. Soc. Hort. Sci. 67:185-189.

Trebitsh, T., J.E. Staub, and S.D. O'Neill. 1997. Identification of a 1amino-cyclopropanel-1- carboxylic acid synthase gene linked to the Female $(F)$ locus that enhances female sex expression in cucumber. Plant Physiol. 113:987-995.

U.S. Department of Agr. 2003. Agricultural statistics. USDA, Natl. Agr. Statistics Serv., Washington, D.C.

Van Ooijen, J.W. and R.E. Voorrips. 2001. JoinMap 3.0, Software for the calculation of genetic linkage maps. Plant Research Intl., Wageningen, The Netherlands.

Vos, P., R. Hogers, M. Beeker, M. Reijans, T. Vandelee, M. Hornes, A. Frijiters, J. Pot, J. Peleman, M. Kuiper, and M. Zabeau. 1995. AFLP-A new technique for DNA fingerprinting. Nucl. Acids Res. 23:4407-4414.

Zamir, D., N. Navot, and J. Rudich. 1984. Enzyme polymorphism in Citrullus lanatus and C. colocynthis in Israel and Sinai. Plant Syst. Evol. 146:163-167.

Zamir, D. and Y. Tadmor. 1986. Unequal segregation of nuclear genes in plants. Bot. Gaz. 147:355-358.

Zhang, R., Y. Xu, K. Yi, H. Zhang, L. Liu, G. Gong, and A. Levi. 2004. A genetic linkage map for watermelon derived from recombinant inbred lines. J. Amer. Soc. Hort. Sci. 129:237-243. 